\title{
Resonant fiber grating devices and their applications
}

\section{Xuewen Shu, Kate Sugden, Ian Bennion}

Xuewen Shu, Kate Sugden, lan Bennion, "Resonant fiber grating devices and their applications," Proc. SPIE 6351, Passive Components and Fiberbased Devices III, 63510A (3 October 2006); doi: 10.1117/12.688919 


\title{
Resonant Fiber Grating Devices and their Applications
}

\author{
Xuewen Shu*, Kate Sugden, Ian Bennion \\ Photonics Research Group, Aston University, Birmingham, B4 7ET, United Kingdom
}

\begin{abstract}
We describe our recent progress in the fabrication and applications of fiber grating based resonant devices including infiber Fabry-Perot etalons and distributed Gires-Tournois etalons.
\end{abstract}

Keywords: fiber Bragg grating, Fabry-Perot etalon, Gires-Tournois etalon, resonant, dispersion

\section{INTRODUCTION}

Fiber grating based devices have attracted considerable research and application interest in the last decade [1]. Fiber grating is a section of optical fiber, in which the refractive index of the core (or even the cladding) has periodical or quasi-periodical variation along its length, scattering light in a way similar to a diffraction grating, and transmitting or reflecting certain wavelengths selectively. Due to its intrinsic all-in-fiber feature, fiber grating devices can directly process optical signals in optical fiber and thus overcome the loss and yield issues associated with traditional bulk-optic devices. In recent years, there are a lot of reported researches on using fiber gratings, especially fiber Bragg gratings (FBG) to generate novel resonant structures. In this paper, we describe our recent progress in the fabrication and applications of fiber grating based resonant devices.

\section{CHARACTERISTICS OF FBG-BASED ETALONS}

(a)

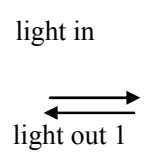

(c)

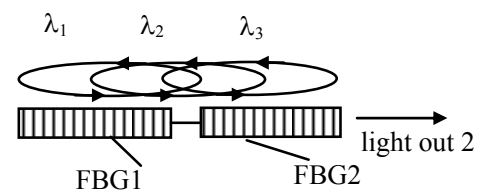

(b)
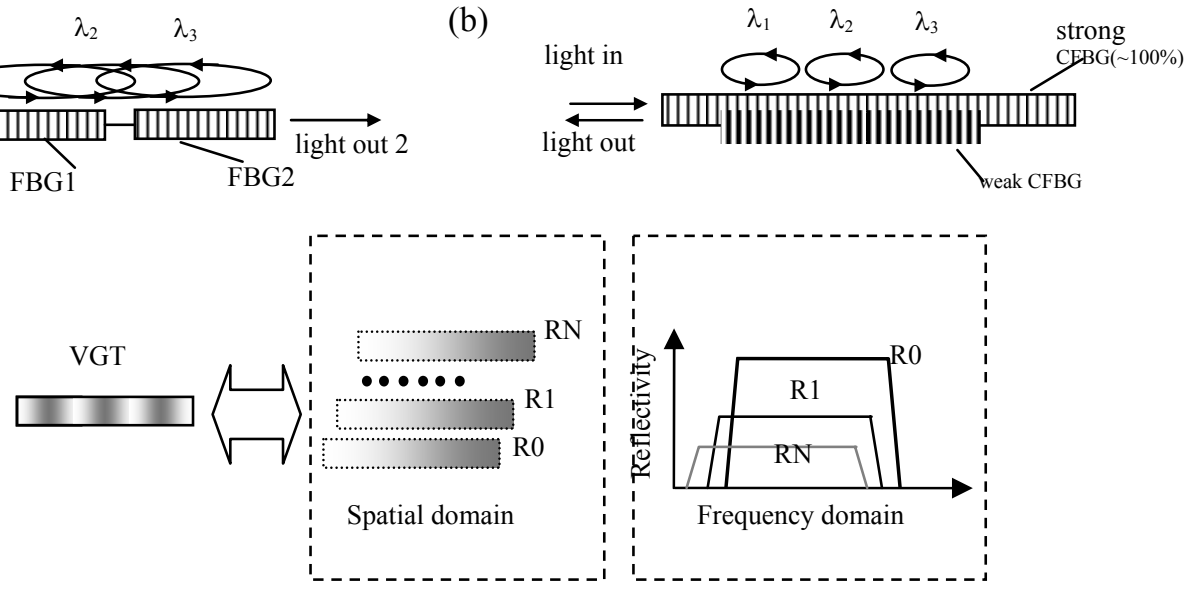

Fig.1 Schematic of typical FBG-based etalon structures. (a)FBG-FPE; (b)DGTE; (c)Virtual GTE

Fig.1 shows achematically serveral typical etalon structures based on fiber Bragg gratings. Fig.1a is a FBG-based FabryPerot etalon (FPE), in which two FBGs are separately located and light resonates between the two gratings. The input light can resonant within the cavity and finally come out to either be reflected or be transmitted. Fig.1b is a FBG-based distributed Gires-Tournois etalon (DGTE), or a special configuration of a FPE, which consists of a partially reflective grating and a highly $(\sim 100 \%)$ reflective grating, in which the strong grating reflects almost all light back to yield the all-

*x.shu@aston.ac.uk, Tel: +44 121 2043549, Fax: +44 1213682

Passive Components and Fiber-based Devices III, edited by

Sang Bae Lee, Yan Sun, Kun Qiu, Simon C. Fleming, lan $\mathrm{H}$. White

Proc. of SPIE Vol. 6351, 63510A, (2006) · 0277-786X/06/\$15 · doi: 10.1117/12.688919

Proc. of SPIE Vol. $635163510 \mathrm{~A}-1$ 
pass transfer function and the first grating is used to control the dispersion properties of the structure. Fig.1c shows the concept of virtual GT etalons (VGTE), which can be generated with complex modulation encoded either in amplitude or in phase. A VGTE is equal to a stack of FBGs in spatial domain and GT resonance is generated within the overlapped frequency range. Before we discuss the characteristics of these etalons, we would first present some theoretical models suitable for simulating these structures.

\subsection{Theoretical models for resonant structures formed by multiple gratings}

The refractive index variation of a structure consisting of multiple fiber Bragg gratings can be described as

$$
n(z)=n_{0}(z)+\operatorname{Re}\left\{\sum_{m} \frac{\Delta n_{m}\left(z-z_{m}\right)}{2} \exp \left[j\left(k_{g 0} \cdot\left(z-z_{m}\right)+\phi_{g}\left(z-z_{m}\right)\right]\right\}\right.
$$

where we have assumed these gratings are displaced each other and have similar profile (i.e. same length, same chirp rate and same apodisation profile) but different modulation amplitudes, $\Delta n_{m} . z_{m}$ is the displacement of the $m$ th grating. For each grating, its central k-vector is $k_{g 0}=2 \pi / \Lambda_{g}, \Lambda_{g}$ is the central grating pitch, $n_{0}$ is the effective average mode index of the optical fiber. The residual phase $\phi_{g}(z)$ describes chirp of the grating and $z$ is the position along the fiber. For a linearly chirped grating, the phase can be described as $\phi_{g}(z)=-C \pi z^{2} /\left(n_{0} \Lambda_{g}^{2}\right)$, where $C$ is the chirp rate.

The electric field of the wave propagation within the structure can be written as

$E=A(z) e^{j \beta z-j \omega t}+B(z) e^{-j \beta z-j \omega t}$

where $A(\mathrm{z})$ and $B(\mathrm{z})$ represent the slowly varying amplitude of the forward and backward modes, respectively. $\beta=2 \pi n_{0} / \lambda$ is the mode propagation constant. The interaction of the forward and backward modes can be described with coupled mode equations [2],

$$
\begin{aligned}
& \frac{d A(z)}{d z}=-j B(z) \sum_{m} k_{m} \exp \left\{j\left[2 \Delta \beta\left(z-z_{m}\right)-\phi_{g}\left(z-z_{m}\right)\right]\right\} \\
& \frac{d B(z)}{d z}=j A(z) \sum_{m} k_{m} \exp \left\{-j\left[2 \Delta \beta\left(z-z_{m}\right)-\phi_{g}\left(z-z_{m}\right)\right]\right\}
\end{aligned}
$$

where $k_{m}=\pi n_{m} /(2 \lambda)$ is the coupling strength of the individual grating, $\Delta \beta=\beta-\pi / \Lambda_{g}$ is the detuning. The transmission and reflection response of the grating can be calculated by solving the above coupled mode equations with boundary conditions $A(0)=1, B(\mathrm{~L})=0$, where $\mathrm{L}$ is the total length of the structure.

More conveniently, one can also use transfer matrix method $[3,4]$ to calculate the spectral response of multiple grating based structures. For each grating, we can divided it into $M$ uniform sub-gratings, with each sub-grating represented by a transfer matrix $\mathrm{F}_{\mathrm{i}}$ with parameters $\Delta \mathrm{L}_{\mathrm{gi}}$ (length), $\Lambda_{\mathrm{i}}$ (period), $\kappa_{\mathrm{i}}$ (coupling coefficient) and $\beta$, which is given by

$$
\mathrm{F}_{i}=\left[\begin{array}{cc}
\cosh \left(\gamma_{i} \Delta L_{g i}\right)-j \frac{\Delta \beta_{i}}{\gamma_{i}} \sinh \left(\gamma_{i} \Delta L_{g i}\right) & -j \frac{\kappa_{i}}{\gamma_{i}} \sinh \left(\gamma_{i} \Delta L_{g i}\right) \\
j \frac{\kappa_{i}}{\gamma_{i}} \sinh \left(\gamma_{i} \Delta L_{g i}\right) & \cosh \left(\gamma_{i} \Delta L_{g i}\right)+j \frac{\Delta \beta_{i}}{\gamma_{i}} \sinh \left(\gamma_{i} \Delta L_{g i}\right)
\end{array}\right]
$$

where $\gamma_{i}=\sqrt{\kappa_{i}^{2}-\Delta \beta_{i}^{2}}$. Note that $\gamma_{\mathrm{i}}$ is imaginary at wavelengths for which $\left|\Delta \beta_{i}\right|>\kappa_{i}$.

Once all of the matrices for the individual sub-gratings are known, the transfer matrix for the whole grating is given by multiplying them together as

$$
\mathrm{T}_{g m}=\mathrm{F}_{M} \cdot \mathrm{F}_{M-1} \cdot \ldots \cdot \mathrm{F}_{i} \cdot \ldots \cdot \mathrm{F}_{1} .
$$

The matrix describing the propagation between two gratings with separation of $d_{m}$ can be written as 


$$
\mathrm{T}_{d m}=\left[\begin{array}{cc}
\exp \left(-j \beta d_{m}\right) & 0 \\
0 & \exp \left(j \beta d_{m}\right)
\end{array}\right]
$$

where $d_{m}=z_{m+1}-z_{m}-L_{g}$. When all the gratings are equally spaced, $d_{m}$ is a constant independent of $m$. Note that we have $d_{m}<0$ when the gratings overlap each other. If the gratings do not overlap each other, then $\mathrm{d}_{m}>0$, which is corresponding to a blank section without refractive index modulation.

The whole structure with multiple gratings can be written as

$$
\mathrm{T}_{\text {total }}=\left[\begin{array}{cc}
T_{1} & T_{2} \\
T_{2}^{*} & T_{1}^{*}
\end{array}\right]=T_{g N} \cdot T_{d N-1} \cdots T_{g 2} \cdot T_{d 1} \cdot T_{g 1}
$$

The complex reflection coefficient and transmission coefficient can be determined by

$$
\begin{aligned}
& r=-\frac{T_{2}^{*}}{T_{1}^{*}} \\
& t=\frac{1}{T_{1}^{*}}
\end{aligned}
$$

Either uniform gratings or chirped gratings can be used to construct etalons. The gratings can also have either separated structure or overlapped structures. All these types of multiple-grating-based etalons can be calculated by Eqn.(8). Also, if $N=2$, Eqn.(8) describes a single-cavity etalon formed by two gratings. If $\mathrm{N}>2$, Eqn.(8) describes a multi-cavity etalon formed by $N$ gratings.

\subsection{Fabry-Perot Etalons}

FBG-based Fabry-Perot etalons (FBG-FPE) have been reported in many literatures [5-16]. FBG-FPE formed by uniform gratings has very limited bandwidth, therefore usually just suitable for narrow band applications [5]. It has been shown that the operation bandwidth can be efficiently expanded when chirped FBGs with overlapped structure are used [6].

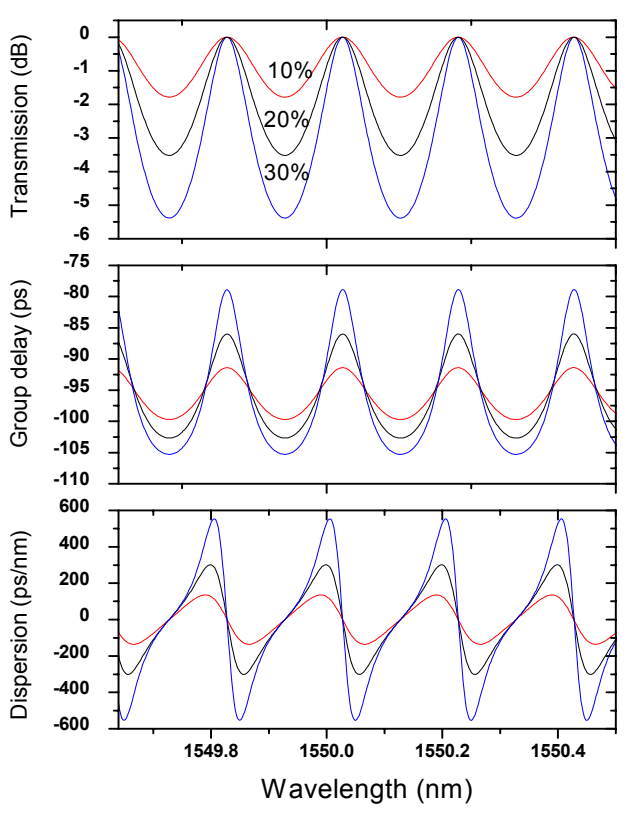

(a)

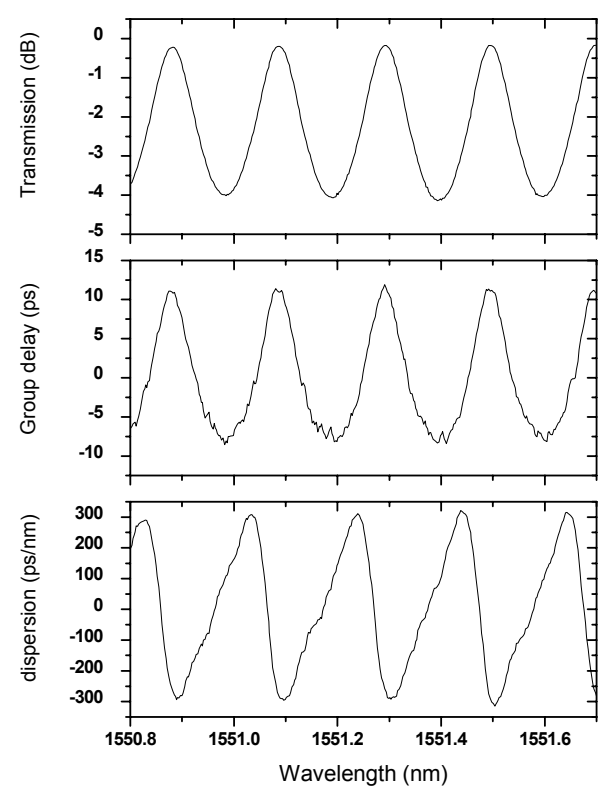

(b)

Fig.2 Spectral response of FBG-FPE. (a)Calculated results for FBG pairs with reflectivity of 10\%, 20\%, 30\%; (c)Experimental results. 
Depending on the reflectivity of the FBGs, the FBG-FPE can have either high finesse [7] or low finesse [6]. High finesse FBG-FPE has applications including DWDM system filtering, multi-wavelength generation and frequency referencing [8-11]. Low finesse FBG-FPE can be used to control dispersion in optical systems since they can be designed to have periodically linear response in dispersion [15,16]. Fig.2a shows the calculated spectral response of transmissive FPE consisting of FBG pairs for the case of different reflectivity. Both gratings have a length of $4 \mathrm{~mm}$ and a chirp rate of $8 \mathrm{~nm} / \mathrm{cm}$. Both gratings are apodised with super-Gaussian profile.The displacement of the two grating is $4.15 \mathrm{~mm}$, which gives the free spectral range (FSR) of $\sim 25 \mathrm{GHz}$. For the reflectivity of $10 \%, 20 \%$ and $30 \%$, the corresponding index modulations are $3.7 \times 10^{-4}, 5.3 \times 10^{-4}$ and $6.8 \times 10^{-4}$, respectively. It is seen in Fig.2a that the amplitudes of group delay peak and the dispersion increase as the reflectivity increasing. The linearity of the dispersion curve degrades with the reflectivity increasing. The transmission loss also varies periodically and increases with increasing FBG reflectivity. Usually, to keep good linearity and reasonable small loss, one may limit the reflectivity to be less than 30\%. Fig.2b shows an experimental example of the measured spectra of a transmissive FBG-FPE. It was formed using a pair of chirped gratings each of length $\sim 4 \mathrm{~mm}$, reflectivity $\sim 22 \%$ and a separation of $\sim 4.1 \mathrm{~mm}$. For each channel, the time delay was quadratic and resulted in linear variation in dispersion, as clearly seen in Fig $2 b$, which agrees well with the theoretical resullts.

\subsection{Gires-Tournois Etalons}

Gires-Tournois etalon (GTE) is a special configuration of a FPE, which consists of a partially reflective mirror (first mirror) and a 100\% reflective mirror (second) [17-21]. It is well known that Gires-Tournois Etalons (GTE) are periodic all-pass filter that can be used to compress ultrashort optical pulses or compensate chromatic dispersion [17-19]. However, tranditional GTEs formed with bulk-optic mirrors or thin film mirrors [17-19] suffer from high insertion loss due to their bulk-optic architecture and alignment and suffer from poor yields in fabrication which increases the manufacturing cost. Therefore, it is desirable if GTE can be directly made in the optical fiber. By using fiber Bragg gratings in DGTEs there is the very attractive advantage of design flexibility. The length, strength, chirp rate and wavelength off-set of the gratings can all be varied to control the dispersion properties, bandwidth and free spectral range of the devices. The fundamental difference between the resonance characteristics of distributed GTE with that of traditional GTE is that for distributed GTEs the resonance takes place in a dispersive cavity, where the different wavelengths resonate at different positions along the structure, while for traditional GTE, the resonance takes place in a largely non-dispersive cavity, where the different wavelengths resonate at same position.

Fig.3 shows some examples of calculated spectral response of DGTEs. In simulation, we assume that each DGTE consisted of two $16 \mathrm{~mm}$-long overlapped chirped FBGs. The chirp rate is $14 \mathrm{~nm} / \mathrm{cm}$ and the displacement of the two

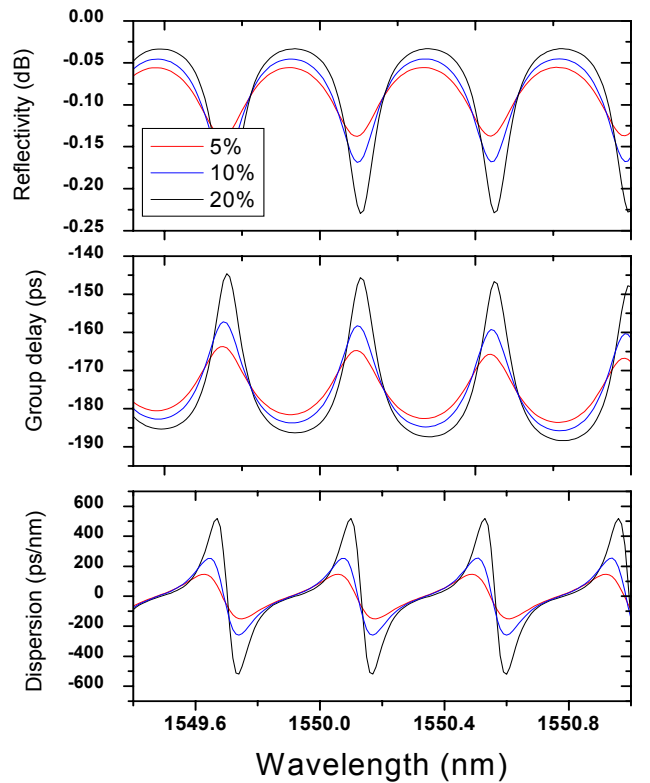

(a)

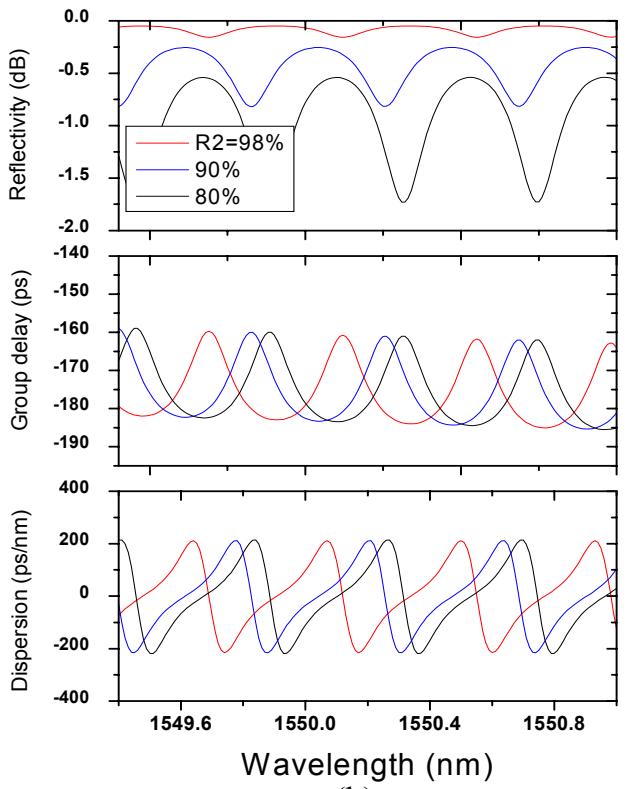

(b)

Fig.3 Calculated spectral response of DGTEs. (a)R2=98\%, R1=5\%, 10\%, 20\%. (b)R1=8\%, R2=98\%, 90\%, 80\%. 
grating is $2.07 \mathrm{~mm}$, which gives FSR of $\sim 50 \mathrm{GHz}$. Fig.3a compares the cases when three DGTEs have same reflecitivity for the strong grating ( $\mathrm{R} 2=98 \%$ ) but different reflectivity $(\mathrm{R} 1=5 \%, 10 \%, 20 \%)$ for the weak grating. It is found that with increasing the reflectivity of the weak grating, the variation of reflectivity increases slightly while the amplitudes the group delay and dispersion increase significantly. The linearity of the dispersion is also degraded with R1 increasing. Fig.3b compares the cases when there DGTE have same reflectivity for the weak grating (R1=8\%) but different reflectivity $(\mathrm{R} 2=98 \%, 90 \%, 80 \%)$ for the strong gratings. It is found that decreasing the reflectivity of the strong grating almost does not change the group delay the amplitudes of the group delay and dispersion but insertion loss and the variation of reflecitivity increasing significantly. Ideally, a DGTE should have the reflectivity of the strong grating close to $100 \%$.

Experimentally distributed GTEs of both separated structure and the overlapped structure have been investigated. Fig. 4a shows the measured reflective response of a DGTE formed by two physically displaced uniform period gratings. The weak grating has a length of $0.2 \mathrm{~mm}$ and reflectivity of $\sim 4 \%$, while the strong grating has a length of $0.8 \mathrm{~mm}$ and reflectivity of $\sim 97 \%$. The separation of the gratings was about $4.1 \mathrm{~mm}$, which resulted in the FSR of $\sim 25 \mathrm{GHz}$, as shown in Fig.4a. The 3dB reflection bandwidth of the DGTE is around $1.9 \mathrm{~nm}$ and the ripples in the reflectivity are less than $0.5 \mathrm{~dB}$ around central band. Similar to the traditional bulk-optic GT, both the time delay and dispersion curves have periodic characteristics over the reflection bandwidth, where nine similar channels can be found in Fig.4a. For each channel, the time delay was quadratic and resulted in linear variation in dispersion, as clearly seen in Fig4a. Despite the similarity, it is interesting to note some spectral difference between bulk-optic GTE and the DGTE based on uniform period gratings. It was found that the group delay of the DGTE shown in Fig.4a has slightly curved trend, where the edge peaks are about 6ps higher than the center peaks. This curved trend is mainly caused by the dispersive properties of the second uniform period grating (since the first grating is much shorter), which is confirmed by calculating the group delay of the grating. The curved trend in group delay thus resulted in dispersion offsets of about $+/-15 \mathrm{ps}$ at the edges (almost neglectable compare with about $+/-580 \mathrm{ps} / \mathrm{nm}$ dispersion variation range). The impact of the dispersion of the uniform period grating on the DGTE can be minimized by shortening the length of the second grating.

Fig. $4 \mathrm{~b}$ shows the measured reflective response of a DGTE formed by two overlapped chirped gratings. The DGTE was realized with a $10 \mathrm{~mm}$-long strong CFBG with a reflectivity of $\sim 98 \%$ and a $6 \mathrm{~mm}$-long weak CFBG with a reflectivity of $\sim 11 \%$. The $50 \mathrm{GHz}$ channel spacing resulted from the relative wavelength shift between the two gratings of the order of

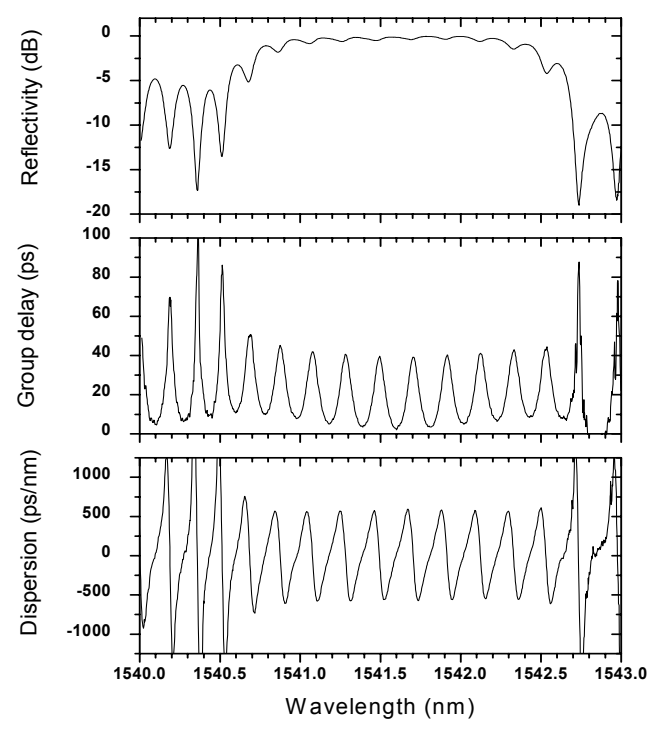

(a)

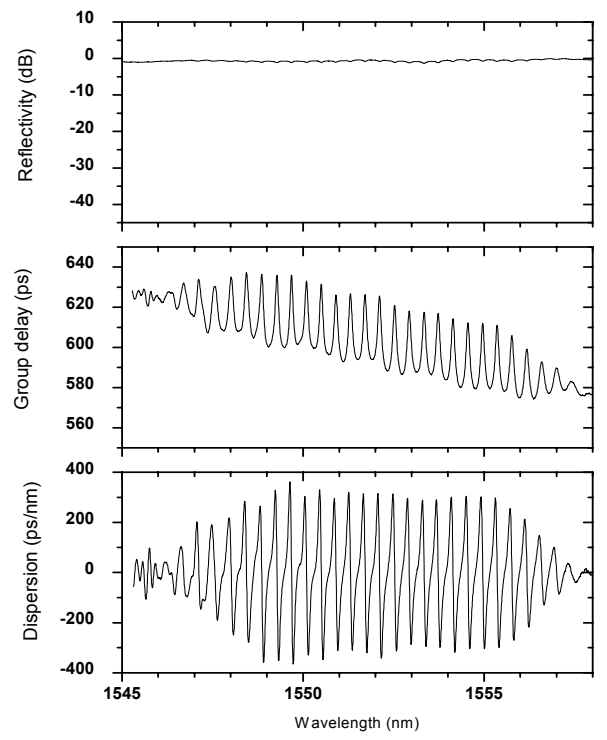

(b)

Fig.4 Measured spectral response of DGTEs. (a) DGTE formed by two separated uniform-period FBGs.

(b) DGTE formed by two overlapped CFBGs.

$2 \mathrm{~mm}$. The DGTE was designed to launch light from long wavelength end. The usable bandwidth of the DGTE is mainly determined by the weak grating with the structure of Fig.1b, which is about 8nm, as seen in Fig.4b. The reflectivity of 
the DGTE is high and the variation in the reflectivity is less than $1 \mathrm{~dB}$, as shown in Fig. $4 \mathrm{~b}$. The CFBG-based DGTEs also have some particular characteristics. It was seen clearly from Fig.4b that the group delay of DGTE has a slope caused by the dispersive nature of the CFBG. This slope in the group delay results in an almost constant offset in dispersion, which is very small (about $-5 \mathrm{ps} / \mathrm{nm}$ ) compared with the variation of dispersion of about $\pm 300 \mathrm{ps} / \mathrm{nm}$. The slope will change to a positive value if the DGTE is design for use with light launched from the short wavelength end. Despite the above difference, the CFBG-based GTE exhibits very similar periodic characteristics to the traditional GTE. The periodic response can be observed in both group delay and dispersion of the DGTE, as shown in Fig.4b. In Fig.4b, the group delay of the DGTE is periodically quasi-quadratic and the dispersion of the DGTE is periodically quasi-linear (for each channel) in a certain bandwidth. It was also noted from Fig.4a and $4 \mathrm{~b}$ that the resonance of FBG-based DGTEs was limited to the reflection band of the CFBGs, which is another different characteristic in spectra compared with traditional GTEs.

Introducing fiber Bragg gratings in DGTEs brings significant advantages in design flexibility. In a GTE, the reflectivity of the first mirror/grating mainly determines the dispersion properties (such as group delay amplitude, dispersion amplitude) of the structure. It is possible to produce arbitrary group delay profiles by tailoring the reflectivity profile of the first mirror/grating - the reflectivity of the second mirror/grating is still kept close to $100 \%$ to achieve all-pass function. For bulk optical mirrors, it is difficult to tailor their reflection spectra and thus such structures would be difficult to achieve. However, by using fiber Bragg gratings in the GTE structure makes the realization of tailored GTE much easier since the reflection spectra of fiber Bragg gratings can be readily tailored during fabrication to have an almost arbitrary profiles [22].

To obtain a target group delay profile, $\Delta \tau(\lambda)$, (i.e. envelop of the group delay amplitude), we derived that the reflection spectrum of the first mirror/grating of the GTE, $R_{I}(\lambda)$, should be tailored to the following profile,

$$
R_{1}(\lambda)=\left[\sqrt{\left[\frac{2 T_{0}}{\Delta \tau(\lambda)}\right]^{2}+1}-\frac{2 T_{0}}{\Delta \tau(\lambda)}\right]^{2}
$$

Where $T_{0}=2 n d / c$ is the round trip time in the cavity, $n$ is the mode effective index, $d$ is the cavity length, $c$ is the light speed in vacuum.

We have fabricated both linearly and nonlinearly tailored DGTEs in the experiments. As examples, three of them are shown in Fig.5. The measured group delay spectra, the envelopes of group delay amplitudes and their corresponding reflection spectra of the first grating recorded in fabrication are plotted in Fig 5a, 5b and 5c, respectively. DGTE (I) and (III) that have FSR of about $50 \mathrm{GHz}$ are formed by two chirped fiber gratings (CFGs) with overlapped structure and DGTE (II) that has FSR of about $25 \mathrm{GHz}$ is formed by two CFGs with separated structure. In fabrication, the second gratings for all these DGTEs have reflectivity of $>95 \%$. It is clearly seen from Fig. 5a and 5b that DGTE (I) has linearly increased amplitude in group delay, while the DGTE (II) and (III) have positive quadratic and negative quadratic varied amplitude in group delay, respectively. Though the oscillation amplitudes of group delay vary channel by channel, for each channel, the group delay is quadratic and result in linear variation in dispersion.
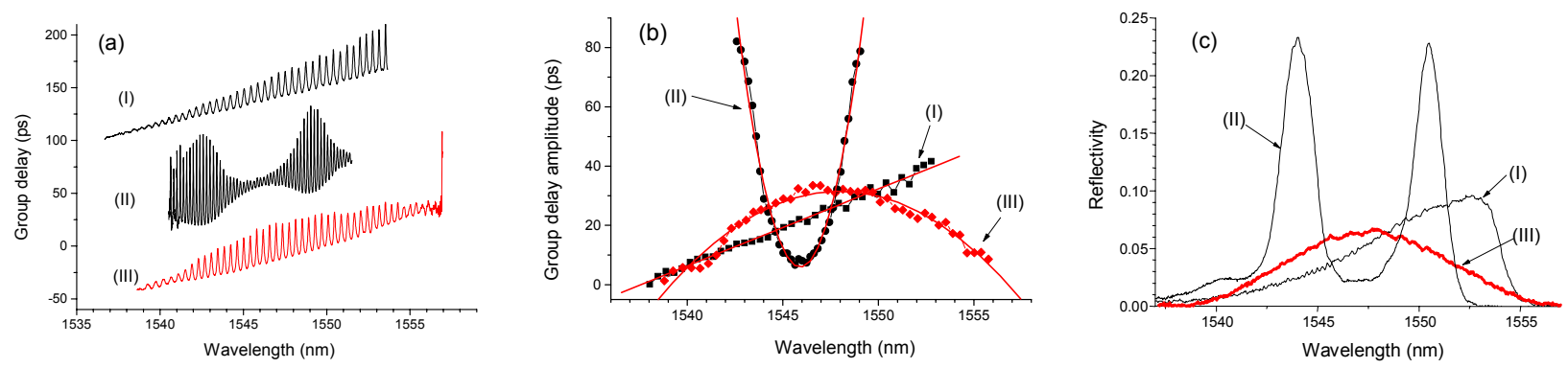

Fig.5. Experimental examples of tailored DGTEs. (a) Measured group delay spectra, (b) the envelopes of group delay amplitudes, (c) reflection spectra of the first grating recorded in fabrication.

\subsection{Single FBG based Virtual Etalons}

Most recently, we proposed and demonstrated a new class of GT etalons, i.e. virtual GT etalons (VGTE) [23,24]. A 
VGTE only need one FBG, which has complex modulation encoded either in amplitude or in phase. A VGTE is equal to a stack of FBGs in spatial domain and GT resonance is generated within the overlapped frequency range.

To create GT resonance with a single FBG, we first consider applying a complex apodisation profile, $F(z)$, along a linearly chirped fiber Bragg grating and the total refractive index variation can be described as

$n(z)=n_{0}+\Delta n \cdot\left[1+F(z) \cdot \cos \left(\frac{2 \pi}{\Lambda(z)} z\right)\right]$

where $\mathrm{n}_{0}$ is the initial refractive index of the optical fiber, $\Delta n$ is the amplitude of the index modulation, $\Lambda(z)$ stands for the linearly varied grating period. $F(z)$ can be expressed as

$$
F(z)=\left[1+\sum_{N} m_{N} \cdot \cos \left(\frac{2 N \pi}{P} z\right)\right] \cdot f(z)
$$

where $m_{N}$ is the coefficient of the $N$ th order cosine modulation, $P$ is the basic period of the cosine function, where $P>>\Lambda$. $f(z)$ is a normal apodisation function, which can be any bell-shape-like one such as super Gaussian function. One can rewrite Eqn.(12) with (13) and obtains

$n(z)=n_{0}+\Delta n \cdot\left[1+f(z) \cdot \cos \left(\frac{2 \pi}{\Lambda(z)} z\right)+\sum_{N} \frac{1}{2} m_{N} \cdot f(z) \cdot \cos \left(\frac{2 \pi}{\Lambda_{+N}(z)} z\right)+\sum_{N} \frac{1}{2} m_{N} \cdot f(z) \cdot \cos \left(\frac{2 \pi}{\Lambda_{-N}(z)} z\right)\right]$

where $\Lambda_{+N}(z)=\frac{P \Lambda(z)}{P+N \Lambda(z)}, \quad \Lambda_{-N}(z)=\frac{P \Lambda(z)}{P-N \Lambda(z)}$.

It is seen from Eqn.14 that the single CFBG with the designed complex apodisation function is equal to a set of overlapped CFBGs that have similar reflection profile (same chirp rate) but different amplitudes (determined by $m_{N}$ ). Though these CFBGs are overlapped each other, they have same chirp rate but different start grating-periods, which are equal to a set of reflectors displaced in spatial domain. The central CFBG reflector can be designed to have reflectivity of as high as to $\sim 100 \%$, which can block interaction between two sides and allows each side to form a GT cavity. Therefore, such an etalon can launch light from both sides.

Fig.6a shows a simulation example of the dual direction GTE. In the simulation, we assume the grating has a length of $14 \mathrm{~mm}$, index modulation amplitude of 0.0024 and a chirp rate of $0.8 \mathrm{~nm} / \mathrm{mm}$. The designed apodisation profile $\mathrm{F}(\mathrm{z})$ has $\mathrm{m}_{1}=0.3, \mathrm{~m}_{2}=\mathrm{m}_{3}=\ldots=\mathrm{m}_{\mathrm{N}}=0, \mathrm{P}=0.173 \mathrm{~mm}$ and $\mathrm{f}(\mathrm{z})$ is the 20 th order super Gaussian function. The generated structure is equal to has three reflectors, which form a single-cavity GT in each side. As expected, the calculated group delay (GD) responses for both launch ends have periodical quadratic oscillations as shown in Fig.6a. It is also seen in Fig.6a that for both launch ends, the resonances have amplitudes of about $27 \mathrm{ps}$ and periods of $50 \mathrm{GHz}$. For comparison, we have also

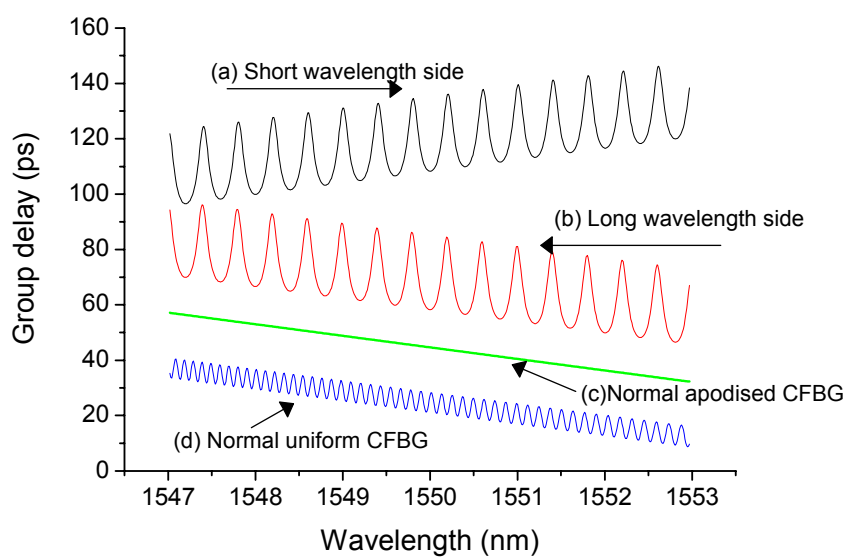

(a)

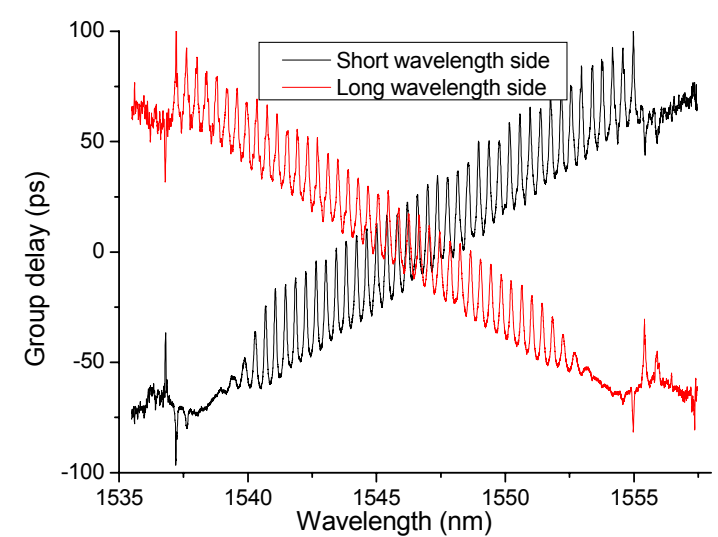

(b)

Fig.6 (a) Calculated group delay response of a dual-direction GT etalon. (I) input from short wavelength end, (II) input from long wavelength end. (III) and (IV) are group delay of a normal apodised CFBG and a normal uniform CFBG, respectively. The data has been offset for clarity. (b) Measured group delay responses from two sides for a dual-direction GT etalon. 
shown in Fig.6a the GD response of a normal super Gaussian apodised CFBG and a uniform CFBG. It is clearly seen in Fig.6a that normal apodised CFBG has no distinct oscillations in group delay and uniform CFBG has small-amplitude non-uniform-period oscillations.

We also fabricated the proposed structure with standard UV inscription technique. A linearly chirped phase mask with chirp rate of $0.84 \mathrm{~nm} / \mathrm{mm}$ was used in the experiment. To write the very fine structure, we reduced the laser beam size with focus lens and aperture to a few tens of micrometers. The apodisation profile was similar to the simulation but $\mathrm{m}_{1}$ was adjusted such that $\mathrm{m}_{1}=0.8$ to compensate non-ideal fringe visibility and $\mathrm{P}=0.33 \mathrm{~mm}$ to ensure channel spacing of $50 \mathrm{GHz}$. The grating had a length of $16 \mathrm{~mm}$, a bandwidth of about $18 \mathrm{~nm}$ and an average transmission depth of about $14 \mathrm{~dB}$. Group delay responses of the fabricated structure from both launch ends were measured and plotted in Fig.6b. As expected, both launch directions show periodical quadratic oscillations in group delays. The amplitudes of the group delay oscillations for the short wavelength launch end and long wavelength launch end were $\sim 35 \mathrm{ps}$ and $\sim 30 \mathrm{ps}$, respectively. The slightly asymmetric response might be due to slight misalignment during the fabrication.

For a VGTE created by phase-modulation, the refractive index variation of the structure can be described as

$$
n(z)=n_{0}(z)+\operatorname{Re}\left\{\frac{\Delta n(z)}{2} \exp \left[i\left(k_{g 0} z+\phi_{g}(z)\right] \cdot \sum_{N} A_{N} \exp \left(i N k_{S} z\right)\right\}\right.
$$

where $k_{S}=2 \pi / P, P$ is the period of the introduced phase modulation. $A_{N}$ is the $N$ th order Fourier coefficient of a phase modulation function $S_{P M}(z)$, i.e.

$$
A_{N}=\frac{1}{P} \int_{0}^{P} S_{P M}(z) \cdot \exp (-i 2 N \pi z / P) d z
$$

Here the phase modulation function can be described as

$$
\begin{aligned}
& S_{P M}(z)=\exp \left[i \phi_{S}(z)\right] \\
& \phi_{S}(z)=\sum_{N} m_{N} \sin \left(N k_{S} z\right)
\end{aligned}
$$

The phase-modulation approach for the VGTE generation is similar to the phase-only sampling method developed for the sampled grating design [25]. However, we require here that the seed grating (chirped fiber Bragg grating) has wide spectral bandwidth so that the generated virtual gratings have overlapped spectral range, where the GT resonance will take place.

Fig.7 shows a design example of the proposed phase-modulation virtual GT etalon. In the simulation, we assume the seed grating has a length of $10 \mathrm{~mm}$, index modulation amplitude of 0.003 , a chirp rate of $12 \mathrm{~nm} / \mathrm{mm}$ and the 20th order super Gaussian apodisation function. The designed phase profile $\phi_{S}(z)$, as shown in Fig. $7 \mathrm{a}$, has $\mathrm{m}_{1}=0.31, \mathrm{~m}_{2}=-0.033$,
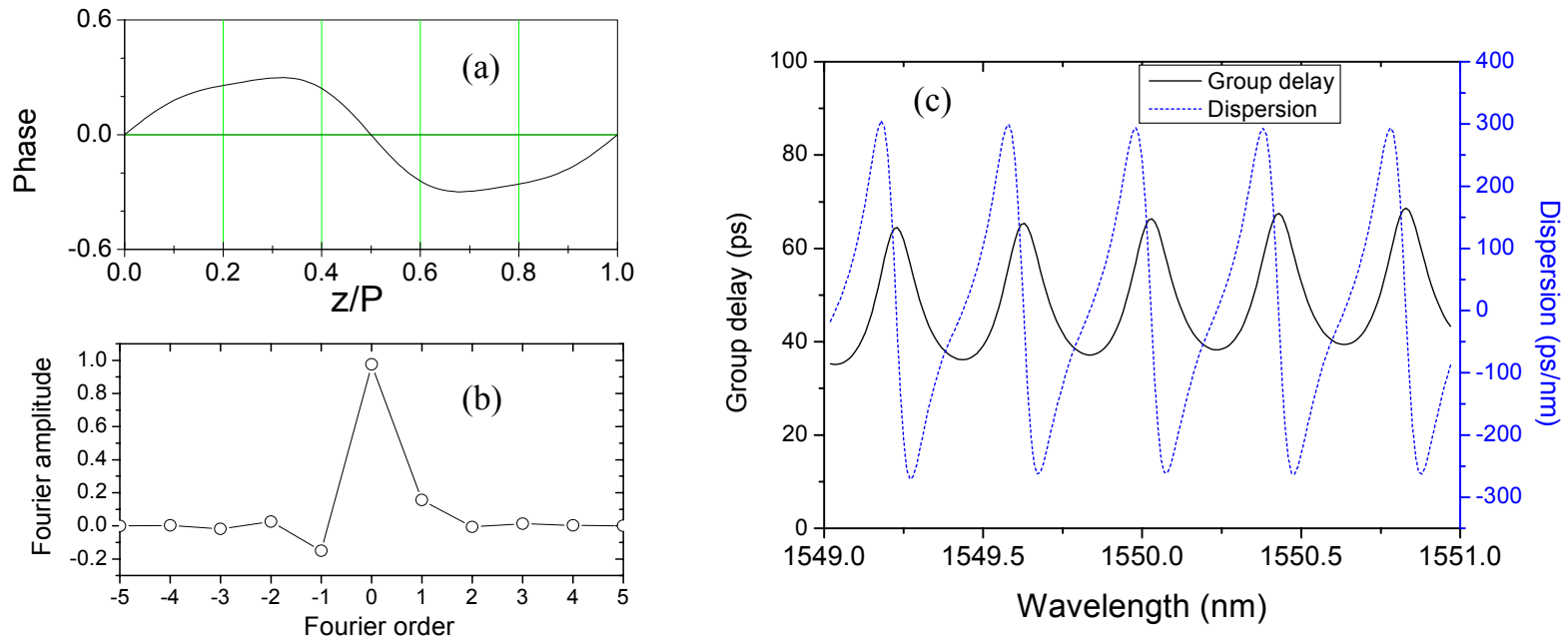

Fig.7 Design of a VGTE with the phase-modulation approach. (a) Designed phase profile in a period, (b) Fourier amplitude of the phase modulation function $\mathrm{S}_{\mathrm{PM}}(\mathrm{z})$, (c) Calculated group delay and dispersion spectra. 
$\mathrm{m}_{3}=0.03, \mathrm{~m}_{4}=\mathrm{m}_{5}=\ldots=\mathrm{m}_{\mathrm{N}}=0, \mathrm{P}=0.115 \mathrm{~mm}$. The Fourier amplitude of the phase modulation function is shown in Fig. $7 \mathrm{~b}$, which indicates generated structure has multiple cavities and also slightly asymmetric for each side. The calculated group delay (GD) and dispersion response from the blue launch end was shown in Fig.7c. As expected, the group delay has periodical quadratic oscillations and the dispersion has periodical linear variation as seen in Fig.7c. The generated VGTE has a FSR of $50 \mathrm{GHz}$, GD resonance amplitude of $28.7 \mathrm{ps}$, and a dispersion variation range of from $-270 \mathrm{ps} / \mathrm{nm}$ to $+300 \mathrm{ps} / \mathrm{nm}$.

A 25-mm-long lithographically prepared phase mask was first fabricated with the phase-modulation pattern superimposed upon an underlying linear chirp with a rate of $15.2 \mathrm{~nm} / \mathrm{cm}$. The test phase mask has simple phase modulation profile with $\mathrm{m}_{1}=0.3, \mathrm{~m}_{2}=\mathrm{m}_{3}=\ldots=\mathrm{m}_{\mathrm{N}}=0$ and a modulation period of $182 \mu \mathrm{m}$, which was designed for $50 \mathrm{GHz}$ FSR. With this phase mask, we then can use our standard UV inscription setup to fabricate etalons. The writing-laser operates at wavelength of $244 \mathrm{~nm}$ and has a beam size of about $1 \mathrm{~mm}$. Several etalons with various length and different strength were fabricated. The measurement results for several etalons were shown in Fig.8. Fig.8a and 8b shows the
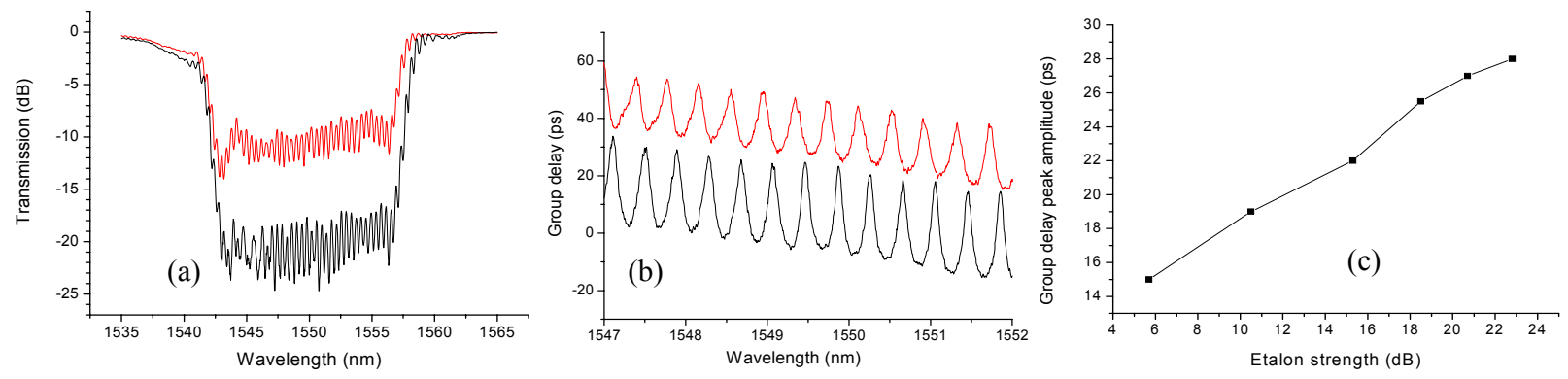

Fig.8 Measurement results for the fabricated VGTEs. (a) Transmission spectra of two VGTEs with different strength. (b) GD response of two VGTEs corresponding to (a). (c) Dependence of GD amplitude on etalon strength.

transmission spectra and group delay response of two etalons generated with the same phase mask. It is seen from Fig.8a and $8 \mathrm{~b}$ that the VGTE with strength of $10.5 \mathrm{~dB}$ has GD oscillation amplitude of $\sim 19 \mathrm{ps}$, while the one with strength of $20.7 \mathrm{~dB}$ has GD oscillation amplitude of $\sim 27 \mathrm{ps}$. By controlling the etalon strength, one may generate etalons with different GD amplitude using the same phase mask, which can be seen more clearly from Fig.8c..

\section{APPLICATIONS}

\subsection{Dispersion compensators or tunable dispersion compensators}

(a)
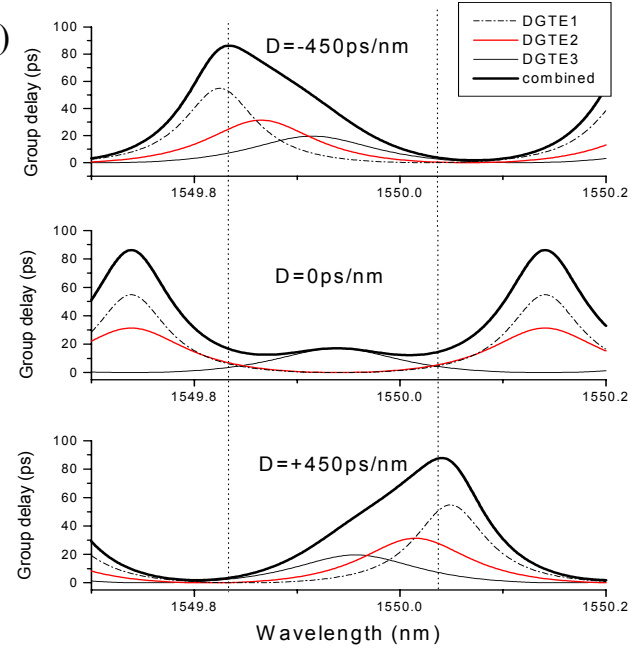

(b)

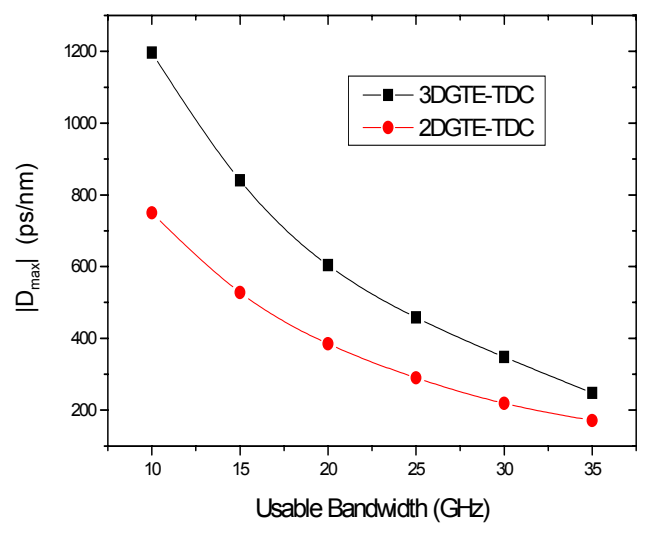

Fig.9 (a) Principle of the operation of a three-DGTE based TDC, (b) dispersion tuning range versus usable bandwidth for group delay ripple of $+/-5$ ps over the full dispersion tuning range. 
Etalons are very suitable for multi-channel dispersion compensation due to their periodical dispersion response. Both FBG-FPE and DGTE can be used to construct dispersion compensators or tunable dispersion compensators $[15,21,26,27]$. Two or more etalons can be combined together to increase the dispersion tuning range and the usable bandwidth. The principle of the operation of tunable dispersion compensator based on three DGTEs can be explained with the simulation results depicted in Fig.9a. The group delay (GD) response for all three DGTEs have same oscillation periods but different amplitudes, which are due to different reflectivity of the weak gratings in these DGTEs chosen. The dispersion of the TDC is determined by the shape of the combined group delay and different dispersion settings are achieved by properly arranging the relative wavelength positions of the group delay peaks of the three DGTEs. For example, negative dispersion is achieved by arranging three GD peaks in a decreasing order around the channel center, while positive dispersion is achieved by arranging them in an increasing order around the channel center as shown in Fig.9a. Zero dispersion is achieved however by setting the smallest GD peak (DGTE3) and other two GD valleys at the channel center as shown in Fig.9a. To minimize the wavelength shift range therefor required temperature change, for a certain channel, we use the shorter wavelength GD peaks to produced negative dispersion and shift to use longer wavelength GD peaks to produce positive dispersion thanks to periodical group delay response of DGTEs. We also investigate the design capability of three-DGTE based TDCs by considering the variables including the FSR, usable bandwidth, the dispersion tuning range and group delay ripples. For a FSR of $50 \mathrm{GHz}$, the dispersion tuning range versus usable bandwidth with group delay ripple of $+/-5$ ps over the full dispersion tuning range is shown in Fig. $9 \mathrm{~b}$, in which that of two-DGTE based TDCs is also shown for comparison. It is clearly seen in the Fig.9b that the relationship between the dispersion tuning range and the usable bandwidth is trade-off, however, three-DGTE based TDCs have much larger tuning range than two-DGTE based TDCs for any designed usable bandwidth. For example, it is seen from Fig. $9 \mathrm{~b}$ that for usable bandwidth of $25 \mathrm{GHz}$, the theoretically expected tuning range of a three-DGTE TDC is about +/$460 \mathrm{ps} / \mathrm{nm}$ while that of a two-DGTE TDC is only about $+/-290 \mathrm{ps} / \mathrm{nm}$. TDC designed with three DGTEs is clearly better than that designed with two DGTEs in increasing the usable bandwidth and dispersion tuning range.

The configuration of a three-DGTE TDC is schematically shown in Fig.10a, which includes a four-port and three-port circulator and three DGTEs with linearly varying dispersion over in excess of a 50nm bandwidth. To achieve the thermal tuning, the three etalons are mounted on individual TECs and then hermetically sealed in a $30.5 \times 56 \times 8.7 \mathrm{~mm}$ butterfly package. Fig.10b shows the resulting group delay of an experimentally constructed three-DGTE TDC with a

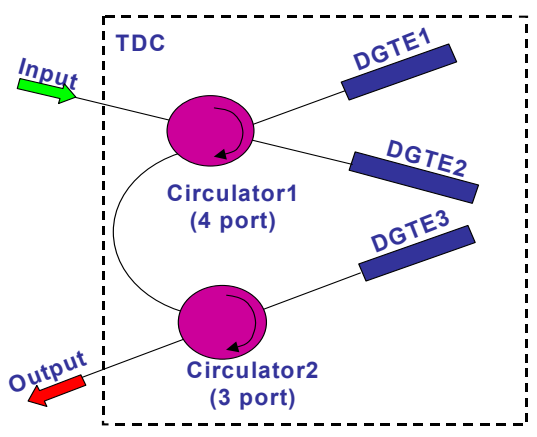

(a)
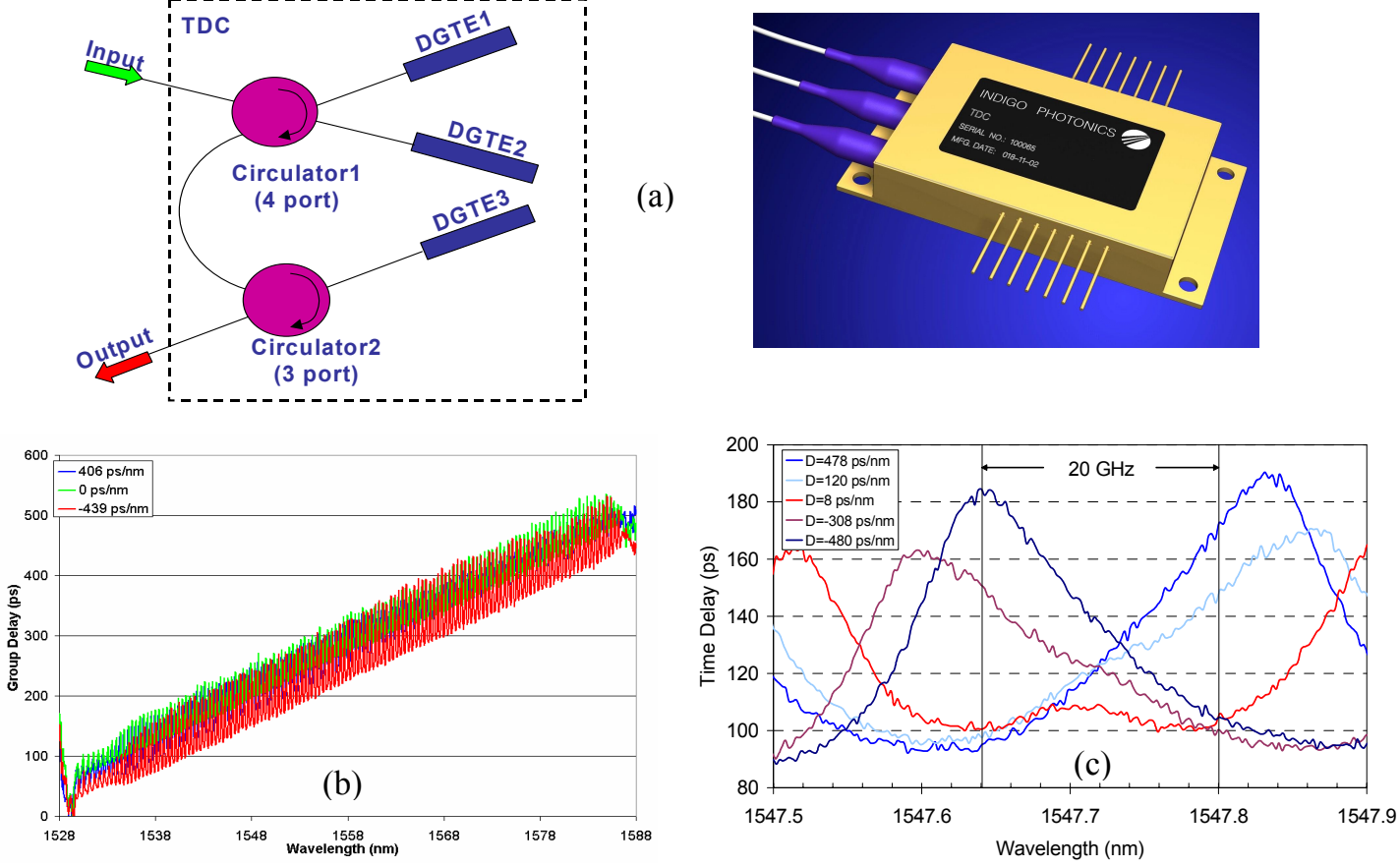

Fig.10 (a) Configuration of TDC based on three DGTEs and the $30.5 \times 56 \times 8.7 \mathrm{~mm}$ package that the etalons fit into., (b) the group delay of the device operating over 120 channels at three dispersion settings and (c) the group delay of a single channel on the $50 \mathrm{GHz}$ grid for different dispersion settings 
channel spacing of $50 \mathrm{GHz}$ over 122 channels for dispersion settings about $-439,0,+406 \mathrm{ps} / \mathrm{nm}$. For clarity, Fig.10c shows the group delay of a single channel on the $50 \mathrm{GHz}$ grid for different dispersion settings. The dispersion level is controlled by the relative temperatures of the three DGTEs. The group delay ripple is $<+/-5 \mathrm{ps}$ over the full dispersion tuning and wavelength ranges. This value is much less than typical CFBG-based TDCs and raises the possibility of cascading such devices through the system (in-line applications), in addition to providing dispersion compensation at the terminal end. A maximum dispersion tuning range of over $+/-500 \mathrm{ps} / \mathrm{nm}$ was achieved with this device over a $20 \mathrm{GHz}$ usable bandwidth. Alternatively $+/-450 \mathrm{ps} / \mathrm{nm}$ tuning range was achieved over a $25 \mathrm{GHz}$ bandwidth. The total insertion loss of the device is about $6.5 \mathrm{~dB}$, which includes about $3.6 \mathrm{~dB}$ loss from the circulators. The PDL of the device is measured to be typically less than $0.2 \mathrm{~dB}$ and the PMD of the device is measured to be typically less than $2 \mathrm{ps}$.

Transmissive FBG-FPEs can be also used to build TDC. Fig.11a show schematically such a configuration, where two transmissive FBG-FPEs are cascaded with an isolator inserted between them to prevent interaction between the two FPEs. The principle of the operation of such a TDC is as follows. FBG-FPE1 and FBG-FPE2 are designed to have positive dispersion slope and negative dispersion slope, respectively. The magnitudes of the slopes are equal (or nearequal) to each other so that there is a region where dispersion is constant. The dispersion value is determined by the relative spectral shift between two FBG-FPEs, which can be controlled either thermally or with strain. The transmissive FBG-FPEs can also work together with reflective DGTEs to form a TDC, which can extend the dispersion tuning range. Fig.11b shows the configuration of a TDC employing two transmissive FBG-FPEs and a reflective DGTE. Experimentally two FBG-FPEs with grating reflectivities of about $20 \%$ were spliced to port 1 and 3 of the optical circulator. The DGTE had grating reflectivity of $10.5 \%$ and $98 \%$ and was spliced to port 2 of the circulator. All the etalons used in the experiment had an equal FSR of $25 \mathrm{GHz}$. Fig. $11 \mathrm{c}$ shows the measured group delay of such a device with dispersion settings at $0,-250,-480,-750$ and $+720 \mathrm{ps} / \mathrm{nm}$. The dispersion tuning range of the TDC was about $+/-$ $800 \mathrm{ps} / \mathrm{nm}$ and the group delay ripples is $+/-4 \mathrm{ps}$ within usable bandwidth of about $13 \mathrm{GHz}$.

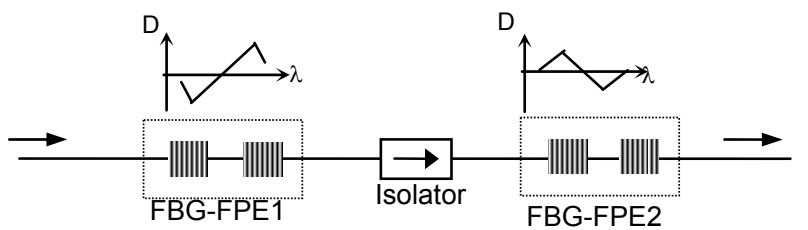

(a)

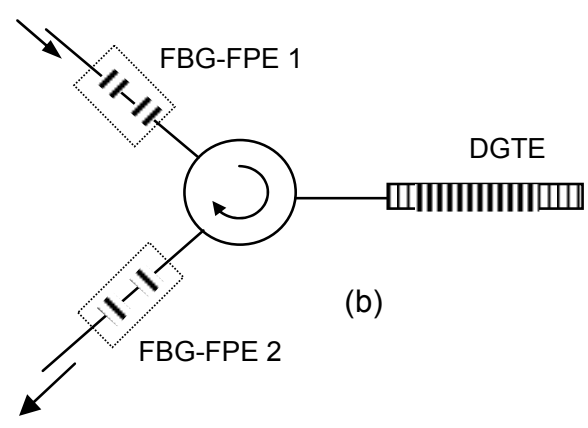

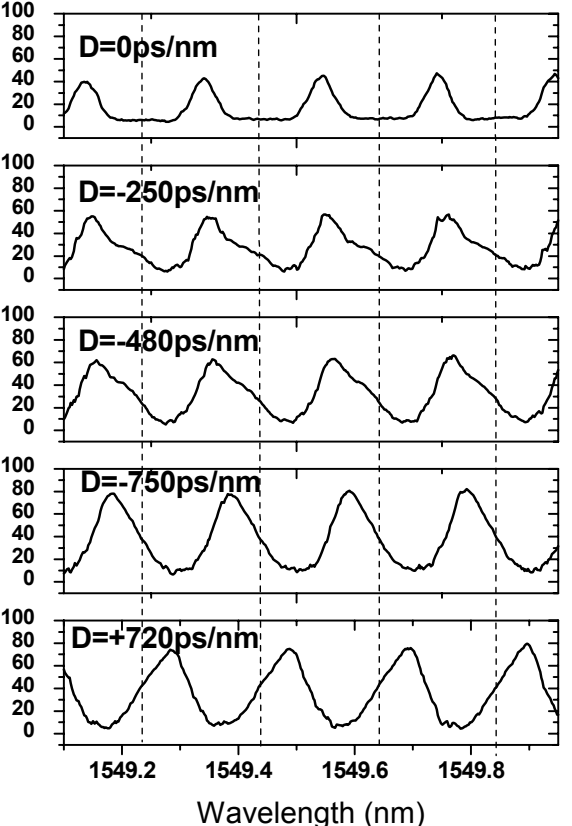

Fig.11 (a) Schematic of the TDC formed by two FBG-FPEs. (b)Schematic of the TDC formed by two FBG-FPEs and a DGTE. (c) Measured group delay of the TDC with configuration of (b) at different dispersion settings

\subsection{Dispersion slope compensators}

High-speed WDM transmission systems also need compensate dispersion slopes. FBG based etalons may also be used for this purpose. We have shown that dispersion slope compensators (DSC) can be realized with two different ways: (1) using FSR-mismatched etalon pair [28]; (2) using tailored DGTEs [22].

Fig.12a and 12b shows the operation principle of dispersion slope compensator based on two DGTEs with different 
group delay amplitude (GDA) and slightly different FSR. In Fig.12a the large-GDA DGTE is designed to have a larger FSR than the small-GDA DGTE. The separation of the group delay peaks of the two DGTEs increases $\left(\Delta \lambda_{1}<\Delta \lambda_{2}<\Delta \lambda_{3}\right)$ as the channel number increases due to the mismatch in FSR as shown in Fig.12a. Since the combined group delay slope, i.e. the dispersion, of the compensator depends on the relative separation between the peaks of the two DGTEs, the dispersion decreases when the separation increases. Over a number of channels the two DGTEs can work together as a negative-slope compensator. However, if we make the large-GDA DGTE have the smaller FSR than that of the smallGDA one, a positive-slope compensator is obtained, as schematically shown in Fig12b. It is clearly shown in Fig. 12b that the group delay slope (i.e dispersion) increases as the relative wavelength separation decrease over the channels $\left(\Delta \lambda_{1}>\Delta \lambda_{2}>\Delta \lambda_{3}\right)$.
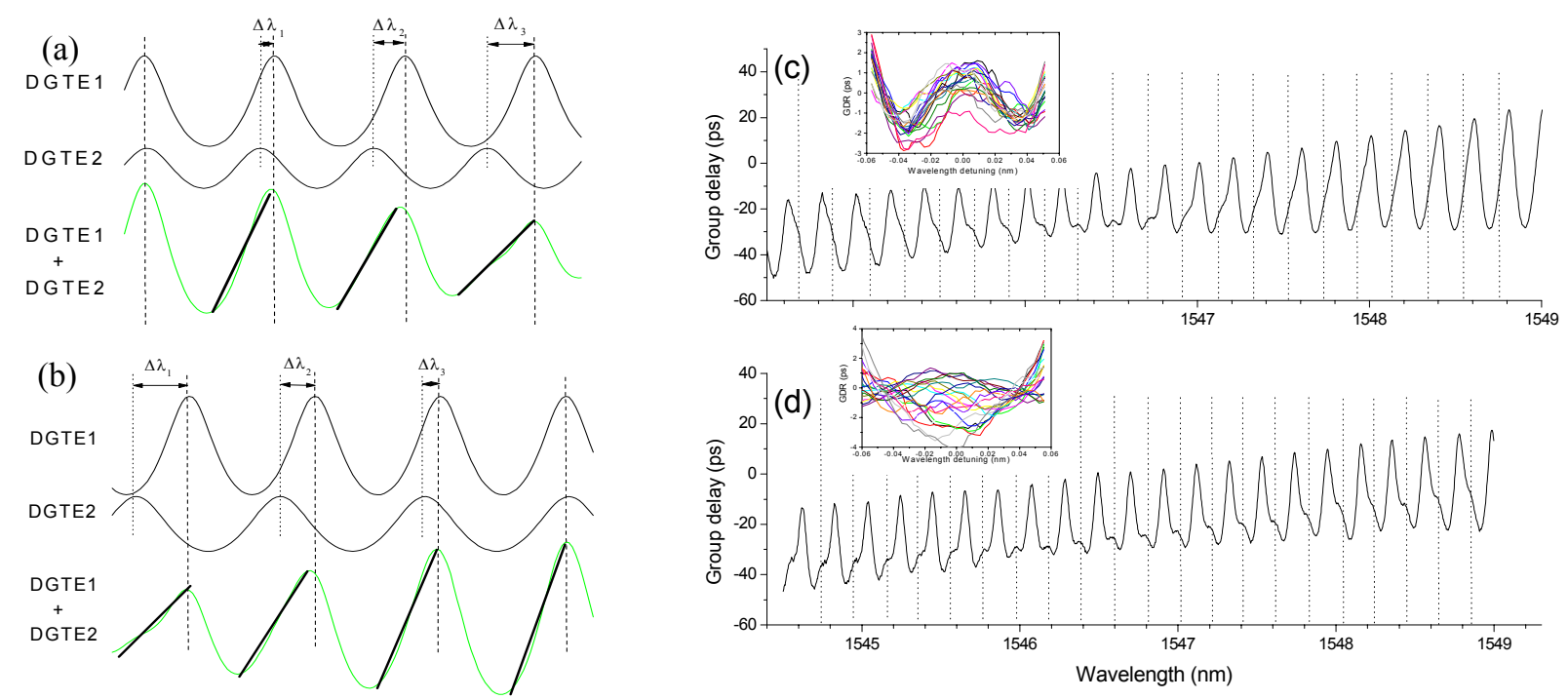

Fig.12 (a)Schamatic of the negative-slope DSC. (b) Schamatic of the positive-slope DSC. (c) Measured group delay of a positive-slope DSC. (d) Measured group delay of a negative-slope DSC.

We experimentally build two DSCs from three DGTEs, namely, DGTE1, DGTE2 and DGTE3, which have FSR of $25.0 \mathrm{GHz}, 25.7 \mathrm{GHz}$ and $26 \mathrm{GHz}$, respectively and their dispersion amplitudes are measured to be $770 \mathrm{ps} / \mathrm{nm}, 260 \mathrm{ps} / \mathrm{nm}$ and 640ps/nm, respectively. The first DSC (DSC1) was built up by cascading DGTE1 and DGTE2 using a 4-port optical circulator. Similarly, the second DSC (DSC2) was built by cascading DGTE2 and DGTE3 through a 4-port optical circulator. Fig.12c and 12d shows the measured group delay response of the two DSCs. It is clearly seen that the first DSC has positive dispersion slope as shown in Fig.12c and the second DSC has negative dispersion slope as shown in Fig. 12d. The dispersion slopes of the DSC1 and DSC2 were $+208.1 \mathrm{ps} / \mathrm{nm}^{2}$ and $-94.9 \mathrm{ps} / \mathrm{nm}^{2}$, respectively.

The principle of combining using two tailored DGTEs as a tunable DSC is schematically shown in Fig.13a. The two DGTEs both have linear envelopes of group delay amplitude over a certain number of channels. The envelopes are properly designed so that for each channel, the dispersion slopes of two DGTEs have same magnitude but different signs in a certain region. Therefor, for each channel, there is a region where dispersion is constant when the two DGTEs are combined. The dispersion value for each channel is determined by the relative spectral shift between two DGTEs, which can be controlled either thermally or by strain. When the dispersion for each channel is set at zero dispersion, as shown in Fig.13a, the dispersion slope over these channels is also zero. When tuning the DGTE1 towards shorter wavelength, the dispersions for all channels increase but the amounts of dispersion changes are different, as shown in Fig.13a, thus a dispersion slope is generated over these channels. The dispersion slope increases as the DGTE1 is shifted further. The group delay spectra of the two linearly tailored DGTEs selected for the experiment are shown in the inset of the Fig.13b. The two DGTEs were combined using a four-port optical circulator. In the experiment, we simply controlled the relative spectral shift between them by applying strain. The measured results for dispersion slope settings of $+20.2,+6.3,-0.7,-$ 9.4, and $-20.2 \mathrm{ps} / \mathrm{nm}^{2}$ for near 30 channels were plotted in Fig.13b, which clearly indicate the slope tunability. The slope 
tuning range of this DSC is from -22 to $+22 \mathrm{ps} / \mathrm{nm}^{2}$ and the usable bandwidth for each channel is about $45 \%$ of the FSR $(50 \mathrm{GHz})$.

To demonstrate even higher order dispersion compensation capability, we designed a DSC using two quadratically tailored DGTEs. The simulated group delay spectra for the two nonlinearly tailored DGTEs are shown in the inset of Fig.13c. The principle of tuning is similar to Fig.13a. When there is a relative spectral shift between each other, the higher order slope (i.e. quadratic coefficients of the dispersion profile) will change. For the plotted dispersion curves in Fig. $13 \mathrm{c}$, the quadratic coefficients vary between +6.3 and -7.1 and one clearly sees the change of dispersion profile over these channels. This example strongly indicates that tunable control of arbitrary dispersion profile may be realized by properly designed tailored DGTEs.

(a)

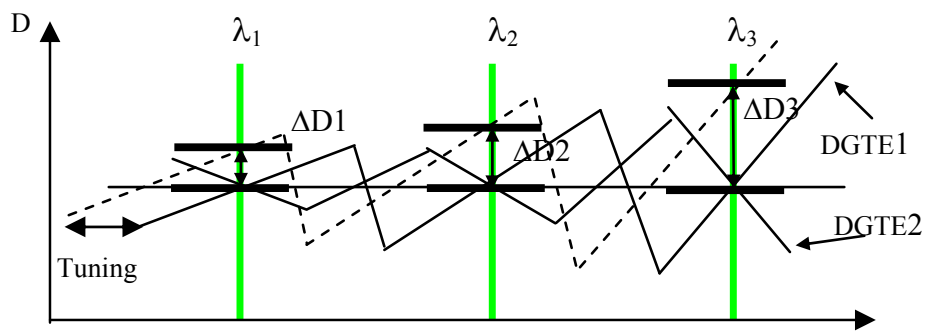

$\lambda$
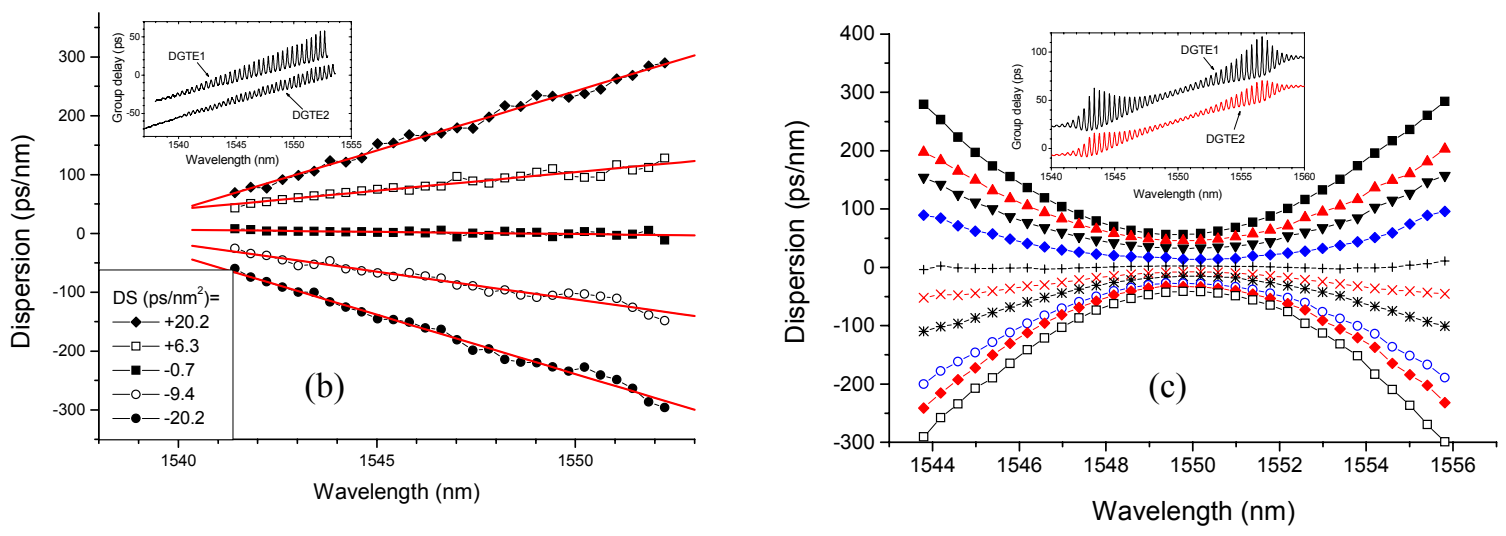

Fig.13 (a) Schamatic of the DSC with tailored DGTEs. (b) Measured dispersion values at different dispersion settings for a.tunable DSC with two linearly tailored DGTEs. inset: measured group delay spectra of the two tailored DGTEs. (c) Design of tunable high-order DSC with two quadratically tailored DGTEs. Inset: calculated group delay spectra of the two tailored DGTEs.

\subsection{Flat-top Multi-passband filters}

Flat top multi-passband filters are very useful in WDM systems, for examples as interleavers [29]. FBG etalons can be used to form all-fiber Michelson-Gires-Tournois interfermeters (MGTIs) and generate flat-top multi-passband profile [30]. The configuration of the all-fiber MGTI filter is schematically shown in Fig.14a, where a CFBG and a DGTE are written in the two arms of a fiber coupler, therefor a Michelson interferometer is formed. The DGTE, acting as phase dispersive mirrors. The reflectivity of CFBG and DGTE are normally chosen to be as high as possible to minimize insertion loss. In a MGTI, the interference inside the DGTE and the interference between light from two arms both exhibit a periodic dependence on the frequency of light, which are determined by the path length difference $(\Delta L)$ of the two arms and the cavity spacing of the DGTE $(d)$, respectively. A proper relationship between them is required to generate desirable transmission function. In general, one can chosen $\Delta L$ to be one half of $d$, i.e. $\Delta L=d / 2$ [29].

In the experiment, the CFBG has a length of $10 \mathrm{~mm}$ and a reflectivity of $\sim 90 \%$. The DGTE was realized with a $10 \mathrm{~mm}-$ long strong grating with a reflectivity of $\sim 90 \%$ and an $8 \mathrm{~mm}$-long weak grating with a reflectivity of $\sim 12 \%$. The relative wavelength shift between the two gratings (i.e. the cavity spacing of DGTE) is about $2 \mathrm{~mm}$. The CFGB and DGTE were spliced to two arms of a $3 \mathrm{~dB}$ fiber coupler to form MGTI according to Fig.14a. The length difference of the two arms 
$(\triangle L)$ was controlled to be around $1 \mathrm{~mm}$ during the splicing. The length difference was then carefully adjusted by
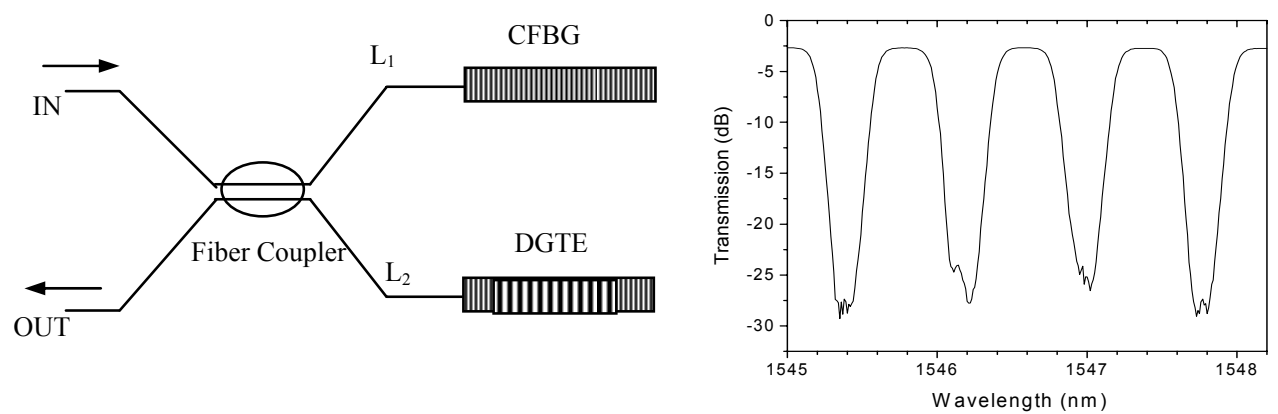

Fig. 14 Configuration (a) and spectral response (b) of an all-fiber MGTI filter.

straining a short section of the fiber in one arm. The spectral response of the MGTI filter was measured using a broadband LED light source and an optical spectrum analyser with a resolution of $0.06 \mathrm{~nm}$. Fig. $14 \mathrm{~b}$ shows its transmission spectrum. It is clearly seen in Fig. $14 \mathrm{~b}$ that the fabricated filter has flattop profile with a channel spacing of $\sim 100 \mathrm{GHz}$. The achieved channel isolation is $23 \mathrm{~dB}$. The passband has square-like shape with the $1 \mathrm{~dB}$ bandwidth of $\sim 40 \mathrm{GHz}$ and $20 \mathrm{~dB}$ bandwidth of $\sim 77 \mathrm{GHz}$, which generate ratio $\mathrm{BW}_{1 \mathrm{~dB}} / \mathrm{BW}_{20 \mathrm{~dB}}$ of $\sim 0.52$. The insertion loss of the filter is $\sim 2.7 \mathrm{~dB}$, which may be expected to be improved by reducing the splicing loss and increasing the reflectivity of the CFBG and DGTE. The configuration shown in Fig.14b may be modified to a two-DGTE based Michelson interferometer, i.e. replacing the CFBG with another DGTE. Such a two-DGTE Michelson interferometer may result in even better square-like passband compared with the demonstrated single-DGTE one [31].

\section{CONCLUSIONS}

FBG-based etalons are very attractive devices and should find many important applications in optical communication systems.

\section{REFERENCES}

[1] K.O. Hill and G. Meltz, "Fiber Bragg grating technology fundamentals and overview", J. Lightwave Technol. 15, 1263-1276 (1997).

[2] H. Kogelnik, "Theory of optical waveguides," in Guided-Wave Optoelectronics, T. Tamir, Ed. New York: SpringerVerlag, (1990).

[3] M. Yamada and K. Sakuda, "Analysis of almost-periodic distributed feedback slab waveguides via a fundamental matrix approach," Appl. Opt., 26, pp. 3474-3478, (1987).

[4] T. Erdogan, "Fiber grating spectra," J. Lightwave Technol. 15, 1277-1294 (1997).

[5]bS. Legoubin, M. Douay, P. Bernage, P. Niay, S. Boj, and E. Delevaque, "Free spectral range variations of gratingbased Fabry-Perot filters photowritten in optical fibers," J. Opt. Soc. Am. A 12, 1687-1694 (1995)

[6] G. Town, K. Sugden, J. Williams, I. Bennion and S. B. Poole, "Wide-Band Fabry-Perot-Like Filters in Optical Fiber," IEEE Photon. Technol. Letters, 7, pp.78-80 (1995).

[7] S. Doucet, R. Slavik and S. LaRochelle, "High-Finesse large band Fabry-Perot fiber filter with superimposed chirped Bragg gratings," Electron. Lett. 38, pp.402-403 (2002).

[8]Y. H. Ja, "Optical vernier filter with fiber grating Fabry-Perot resonators," Appl. Opt. 34, pp.6164-6167 , (1995)

[9] R.J. Forster, N. Langford, A. Gloag, L. Zhang, J.A.R. Williams, I. Bennion, "Longitudinal mode control of an erbium ring fibre laser containing an intracavity chirped Bragg grating etalon," Opt. Commun. 141, pp.283-287 (1997)

[10] G. Brochu, R. Slavik and S. LaRochelle, "Ultra-Compact $52 \mathrm{~mW}$ 50-GHz spaced 16 channels narrow-line and single polarization fiber laser," in Optical Fiber Communication Conference (The Optical Society of America,

Washington, DC, 2004), postdeadline paper PDP22.

[11] J. Azaña, P. Kockaert, R. Slavík, L. R. Chen and S. LaRochelle, "Generation of a 100-GHz Pulse Train by Repetition Rate Multiplication of a 10-GHz Mode-Locked Optical Pulse Sequence using Superimposed Fiber Bragg 
Gratings", IEEE Photon. Technol. Lett., 15, pp. 413-415 (2003)

[12] R. Slavik, S. Doucet and S. LaRochelle, "High-Performance all-fiber Fabry-Perot filters with superimposed chirped Bragg gratings," J. Lightwave Technol. 21, 1059-65 (2003).

[13] C. Sung-Hak, I Yokota and M. Obara, "Free spectral range variation of a broadband, high-finesse, multi-channel Fabry-Perot filter using chirped fiber Bragg gratings," Jpn. J. Appl. Phys. Part 1, 36, pp.6383-6387 (1997).

[14] S. Pereira and S. LaRochelle, "Field profiles and spectral properties of chirped Bragg grating Fabry-Perot interferometers," Opt. Express, 13, 1906-1915 (2005)

[15] X. Shu, K. Sugden, "Transmissive in-fiber Fabry-Perot etalons as a tunable dispersion compensator and a dispersion slope compensator," Opt. Lett. 28, pp.1897-1899 (2003)

[16] J. Zhang, P. Shum, N.Q. Ngo, S.Y. Li, X.P. Cheng,X.F. Yang, J.H. NgA,"FBG etalon in transmission based tunable dispersion slope compensator,", Opt. Commun., 241, pp. 113-119, (2004).

[17] J. Kuhl, and J. Heppner, " Compression of femtosecond optical pulses with dielectric multilayer interferometers,"IEEE Trans. Quantum Electron. QE22, 182-185 (1986)

[18] A.H. Gnauck, L.J. Cimini, J. Stone, and L.W. Stulz, " Optical equalization of fiber chromatic dispersion in a 5Gb/s transmission system," IEEE Photon. Technol. Lett. 2, 585-587 (1990).

[19] D.J. Moss, M. Lamont, S. McLaughlin, G. Randall, P. Colbourne, S. Kiran, and C.A. Hulse, "Tunable dispersion and dispersion slope compensators for $10 \mathrm{~Gb} / \mathrm{s}$ using all-pass multicavitiy etalons," IEEE Photon. Technol. Lett. 15, pp.730-732, (2003).

[20] X. Shu, K. Sugden, K. Byron, "Bragg grating-based all-fiber distributed Gires-Tournois etalons,” Opt. Lett., 28, pp.881-883, (2003).

[21] S. Doucet, R. Slavík, and S. LaRochelle, Tunable Dispersion and Dispersion Slope compensator Using Novel Gires-Tournois Bragg Grating Coupled-Cavities, IEEE Photon. Technol. Lett., 16, 2529-2531, (2004).

[22] X. Shu, I. Bennion, J. Mitchell, and K. Sugden, "Tailored Gires-Tournois etalons as tunable dispersion slope compensators," Opt. Lett. 29, pp.1013-1015, (2004)

[23] X. Shu, K. Sugden, I. Bennion, "All-fiber dual-direction Gires-Tournois etalon realized with single complex fiber Bragg grating structure”, ECOC'2005, Tu4.6.7, (2005)

[24] X. Shu, K. Sugden, I. Bennion, "Virtual distributed Gires-Tournois etalon based on phase-modulated wideband chirped fiber grating", OFC2006, paper OFF4.

[25] J. E. Rothenberg, H. Li, Y. Li, J. Popelek, Y. Sheng, Y. Wang, R. B. Wilcox, and J. Zweiback, "Dammann Fiber Bragg Gratings and Phase-Onlys ampling for High Channel Counts", IEEE Photon. Technol. Lett., 14, pp.1309-1311 (2002).

[26] X. Shu, K. Sugden, P. Rhead, J. Mitchell, I. Felmeri, G. Lloyd, K. Byron, Z. Huang, Igor Khrushchev and I. Bennion, "Tunable Dispersion Compensator Based on Distributed Gires-Tournois Etalons," IEEE Photon. Technol. Lett. 15, pp.1111-1113 (2003).

[27] X. Shu, K. Chisholm, J. Mitchell, I. Felmeri, P. Rhead, A. Gillooly, K. Sugden, I. Bennion., "Tunable dispersion compensator based on three distributed Gires-Tournois etalons", Opt. Comm. 251, pp.59-63 (2005)

[28] X. Shu, K. Chisholm, K. Sugden, "Design and realization of dispersion slope compensators using distributed GiresTournois etalons," IEEE Photon. Technol. Lett. 16, pp.1092-1094, (2004)

[29] Dingel BB, Aruga T, "Properties of a novel noncascaded type, easy-to-design, ripple-free optical bandpass filter," J. Lightwave Tech., 17, pp.1461-1469. (1999)

[30] X. Shu, K. Sugden and I. Bennion, "Novel multipassband optical filter using all-fiber Michelson-Gires-Tournois structure", IEEE Photon. Tech. Lett. 17, pp.384-386, (2005)

[31] Hsieh CH, Wang RB, Wen ZQJ, McMichael I, Yeh P, Lee CW, Cheng WH, "Flat-top interleavers using two GiresTournois etalons as phase-dispersive mirrors in a Michelson interferometer," IEEE Photon. Technol. Lett., 15, pp.242$245,(2003)$ 